\title{
Determining the Ages of Nearby A-Stars with Long-Baseline Interferometry
}

\author{
Jeremy Jones $^{1}$, R. J. White ${ }^{1}$, T. Boyajian ${ }^{2}$, G. Schaefer ${ }^{1}$, E. Baines ${ }^{3}$, \\ M. Ireland ${ }^{4}$, J. Patience ${ }^{5}$, and The CHARA Team ${ }^{1}$ \\ ${ }^{1}$ Georgia State University, ${ }^{2}$ Yale University, ${ }^{3}$ Naval Research Laboratory, ${ }^{4}$ Australian National \\ University, ${ }^{5}$ Arizona State University \\ email: jones@astro.gsu.edu
}

\begin{abstract}
We determine the age of 7 stars in the Ursa Major moving group using a novel method that models the fundamental parameters of rapidly rotating A-stars based on interferometric observations and literature photometry and compares these parameters (namely, radius, luminosity, and rotation velocity) with evolution models that account for rotation. We find these stars to be coeval, thus providing an age estimate for the moving group and validating this technique. With this technique validated, we determine the age of the rapidly rotating, directly imaged planet host star, $\kappa$ Andromedae.
\end{abstract}

Keywords. techniques: interferometric, stars: early-type, stars: evolution, stars: individual ( $\kappa$ And), stars: rotation

\section{The Age of the Ursa Major Moving Group}

We use the Classic, CLIMB, and PAVO beam combiners on the CHARA Array to measure the interferometric visibilities of 7 stars in the Ursa Major moving group. By comparing the measured visibilities and photometry taken from the literature to modeled visibilities and photometry which takes rapid rotation into account, we determine various fundamental parameters of the stars, including radius, luminosity, and rotation speed. We compare these properties to MESA evolution models to determine ages for each of these stars and find an average age for 6 of the stars (excluding the outlier, 59 Dra) of $438 \pm 14 \mathrm{Myr}$, in agreement with previous age estimates (e.g., King et al. 2003). These results are presented in Jones et al. (2015) and below in Fig, 1.

\section{2. $\kappa$ And: A Directly Imaged Planet-Host Star}

$\kappa$ Andromedae is a rapidly rotating A-star and is host to a substellar companion discovered through direct imaging by Carson et al. (2013). Using the technique validated with the Ursa Major moving group, we find an age of 27 Myr for the system assuming solar metallicity, implying that the companion is of planetary mass. Figure 2 shows $\kappa$ And's position on an HR-Diagram.

\section{References}

Boyajian, T. S., et al. 2012, ApJ, 745, 101

Caffau, E., et al. 2011, $S o P h, 268,255$

Carson, J., et al. 2013, ApJ, 763, 32

Jones, J., et al. 2015, ApJ, submitted

King, J. R., et al. 2003, AJ, 125, 1980 


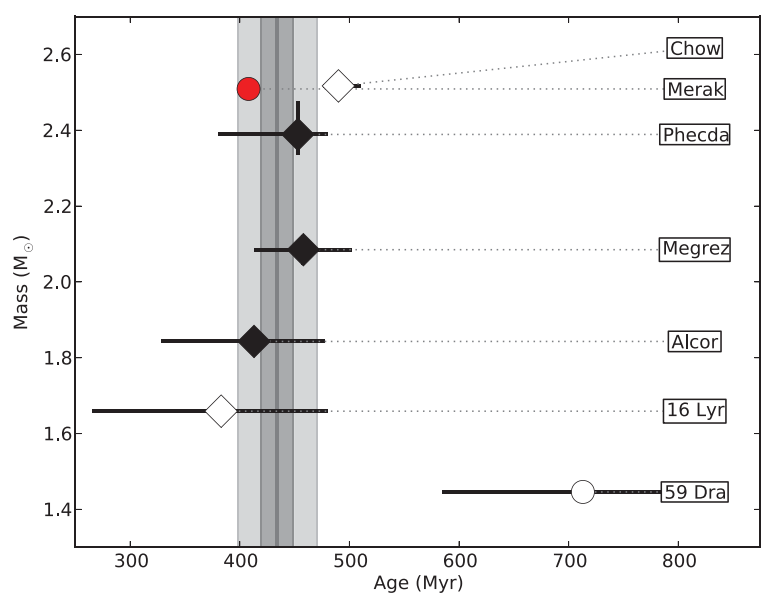

Figure 1. Distribution of stellar masses versus age for 7 stars in the Ursa Major moving group. The circles are slowly rotating stars $\left(V_{\mathrm{e}}<170 \mathrm{~km} \mathrm{~s}^{-1}\right)$ and the diamonds are rapidly rotating $\left(V_{\mathrm{e}}>170 \mathrm{~km} \mathrm{~s}^{-1}\right)$. The black points are nucleus members and the white points are stream members. The red point shows the mass and age of the nucleus member, Merak, that was previously observed by Boyajian et al. (2012). The dark vertical lines represent the mean ages, the broad shaded regions represent the standard deviation of ages, and the narrow shaded regions represent the the standard error in the mean. The age of the outlier, 59 Dra, was excluded when determining the mean, standard deviation, and standard error in the mean.

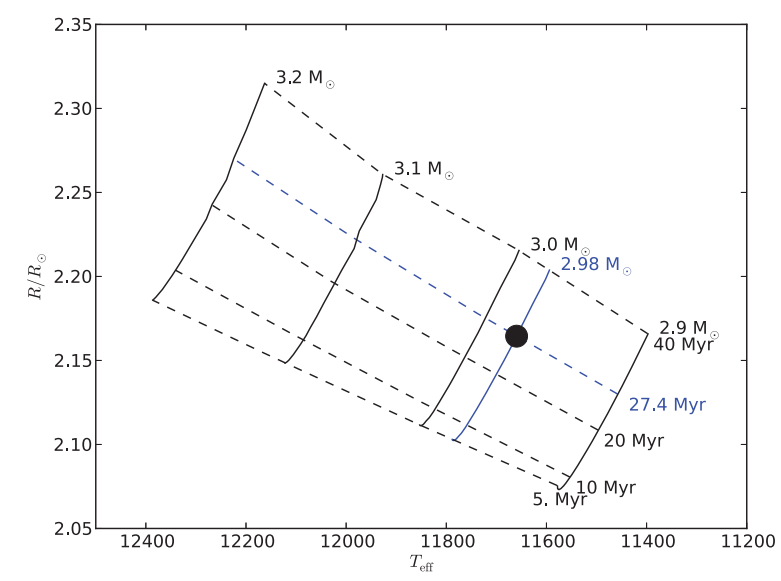

Figure 2. HR-Diagram showing (the black circle) average radius and temperature of the directly imaged planet host star, $\kappa$ Andromedae, compared to evolutionary mass tracks (solid lines) and isochrones (dashed lines) from the MESA evolutionary models which take stellar rotation into account. The blue mass track and isochrone are linear interpolations based on these.

\section{Discussion}

QUESTIONER: You say you use solar metallicity for your age determination for $\kappa$ And. Which solar metallicity?

JONES: For this work, we used $\mathrm{Z}=0.02$.

Questioner: You should use the new solar, $\mathrm{Z}=0.0153$, from Caffau et al. (2011).

Jones: With $\mathrm{Z}=0.0153$ and the previously determined radius and temperature, I find an age for $\kappa$ And of 75 Myr. However, since the previously determined parameters are affected by the mass, this is preliminary! 